\title{
Optimal Control of Switched Homogeneous Systems
}

\author{
Michael Rinehart* , Munther Dahleh*, and Ilya Kolmanovsky ${ }^{\dagger}$ \\ *Dept. of Elec. Eng. and Comp. Sci., Mass. Inst. of Tech., Cambridge, MA, Emails: mdrine@mit.edu, dahleh@mit.edu \\ ${ }_{\dagger}^{\dagger}$ Ford Research and Adv. Eng., Ford Motor Co., Dearborn, MI., Email: ikolmano@ford.com
}

\begin{abstract}
In this paper, we present a method for designing discrete-time state-feedback controllers for a class of continuous-time switched homogeneous systems which includes switched linear systems as a special case. A discrete-time approximate value iteration over a quantization of the unit sphere is used to compute an approximation of the continuoustime value function over the entire unbounded state space. Properties of the value function and its approximations are elicited and used to provide conditions under which statefeedback controllers with provable guarantees in stability and performance can be constructed. To illustrate the results, the methodology is applied to an example switched system possessing two unstable modes, one of which is nonlinear.
\end{abstract}

\section{INTRODUCTION}

In this paper, we examine an optimal control problem for a class of switched homogeneous systems. The systems in this class may be viewed as a subclass of hybrid systems in which the operating mode is treated as an external input and each subsystem possesses homogeneity properties. We concern ourselves with optimizing the performance of the system by designing a state-feedback switching law that approximately minimizes a cost function, which also satisfies certain homogeneity properties. The problem formulation covers, as special cases, switched linear systems and nonlinear switched systems for which accurate homogeneous approximations can be developed. To determine an approximately-optimal control law, we compute an approximation to the value function over the unit sphere, which, by homogeneity, can be extended to provide an approximation to the value function over the entire state space.

One important aspect to our work is the use of dynamic programming to compute an approximately optimal control law. A novel approach to approximating the value function of a discrete-time switched linear system is presented in [1], where approximate value iteration over a finite subset of a complete function basis is used to perform the approximation. Computational complexity is easily managed by scaling the number of functions in the subset, and, under certain conditions, the algorithm is guaranteed to produce a stabilizing feedback law. However, the results rely on the existence of a function in the finite subset that satisfies certain bounds over the state space, which is not guaranteed.

In [2], a slightly different approximate value iteration is used to approximate the value function. For certain classes of systems, the methodology leverages the structure of the finite-horizon value function as the minimum over a finite set of functions. The resulting controller arising from this approximate value function is guaranteed to be stabilizing and approximately optimal, but for more general systems or costs functions, it is not clear if the algorithm is tractable. In this case, a quantization of the state-space may be considered, but the benefits in [2] may no longer apply under a quantization.

Beyond switched linear systems, the work in [3] offers a constructive proof of the existence of stabilizing discretetime feedback controllers for a class of asymptotically controllable continuous-time homogeneous systems. Furthermore, it provides an algorithm for computing an approximation to the feedback law. However, when the results of [3] are specialized to switched homogeneous systems, the assumption on homogeneity requires each subsystem of the switched system to have the same homogeneity characteristics, including degree.

In this work, we leverage homogeneity to reduce the computation of an approximation to the continuous-time value function over the unbounded state space to a linear program over a finite set. We also provide conditions under which a discrete-time feedback law exists and is stabilizing and approximately optimal. Furthermore, it is not necessary for the subsystems to have the same degree of homogeneity. This work may be seen as a generalization of the approach we used in [4], which applied to controllable, degree-1 homogeneous systems.

This paper is organized as follows. In sections II and III, we lay out basic definitions and assumptions and also provide some rudimentary results concerning dynamic programming. In section IV, we prove the existence of a sampling time that generates an asymptotically controllable discrete-time system. Finally, in section V, we introduce an algorithm (a value iteration over a finite subset of the unit sphere) that computes a discrete-time state-feedback controller with guarantees in stability and performance. We provide an example of the methodology applied to a switched system consisting of an unstable degree- 1 and an unstable degree- 3 subsystem. All proofs for the results in this paper may be found in [5].

\section{BACKGROUND}

\section{A. Continuous-Time (CT) Switched Systems: Definitions and Notations}

We consider the problem of stabilizing CT switched systems of the form

$$
\dot{y}(\tau)=g_{i(\tau)}(y(\tau))
$$


where $y(\tau) \in \Re^{n}$ is the state, $i(\tau) \in Q$ is the switching input and is a piecewise-constant function continuous from the right, and $Q \subset Z^{+}$is the set of modes and is a finite set. Because $i$ is continuous from the right, the one-sided limit of $i$ at $\tau$,

$$
i\left(\tau^{-}\right)=\lim _{\alpha \rightarrow \tau, \alpha<\tau} i(\alpha)
$$

may not be equal to $i(\tau)$. In fact, at points of discontinuity (a switch), the two will not be equal.

At times, we want to explicitly express the trajectory of (1) as a function of time, the initial condition, and the input $i$. Denote the value at time $\tau$ of the trajectory originating from $y_{0}$ under a switching law $i$ as $y\left(\tau, y_{0}, i\right)$.

We treat $i$ as a design parameter for the system and seek a feedback control law that stabilizes (1). To this end, we focus our attention to the class of switched systems that can be controlled to the origin in the following formal sense [6].

Definition 1: System (1) is asymptotically controllable if:

1) (attractiveness) for each $y_{0}$ there exists a switching law $i$ such that $y\left(\tau, y_{0}, i\right) \rightarrow 0$ as $\tau \rightarrow \infty$.

2) (Lyapunov stability) for each $\epsilon>0$ there exists $a \delta>0$ such that for each $\left\|y_{0}\right\|<\delta$ there exists a switching law $i$ as in 1) such that $\left\|y\left(\tau, y_{0}, i\right)\right\|<\epsilon$.

3) (no Zeno effect) $i$ has a finite number of switches in a finite time interval.

We now define several important notations used throughout the paper:

- let $\tau_{0}=0$ and successively define the $k^{\text {th }}$ switching instance $\tau_{k}$ as the first time $i(\tau)$ changes value since time $\tau_{k-1}$, i.e. $\tau_{k}=\min _{\tau>\tau_{k-1}}\left\{\tau \mid i\left(\tau^{-}\right) \neq i(\tau)\right\}$

- denote the dwell time of the $k^{\text {th }}$ switch as $\Delta_{k}=\tau_{k+1}-$ $\tau_{k}$,

- define $y_{k}=y\left(\tau_{k}\right)$ as the $k^{\text {th }}$ switching state,

- and define $i_{k}=i\left(\tau_{k}\right)$ as the $k^{\text {th }}$ operating mode and denote the mode sequence as the list $\left(i_{0}, i_{1}, \ldots\right)$.

If the mode becomes a constant after some switching time $t_{k}$, i.e. $i(\tau)=a$ is constant for $\tau \geq \tau_{k}$, then as there are no more switches, we define $\tau_{j}=\infty$ and $i_{j}=a$ for all integers $j \geq k$.

At times, we equivalently express $i$ by its mode and dwell time sequence $\left(i_{k}, \Delta_{k}\right)_{k}$, which will be useful for expressing the dynamics of (1) between switching instances.

\section{B. Assumptions}

In this paper, we consider CT switched systems possessing certain homogeneity properties, which will significantly reduce the difficulty of computing a stabilizing control law. Below is the definition of homogeneity used in this paper.

Definition 2: A function $h$ is degree- $(d+1)$ homogeneous-in-the-state if there exists a matrix function $G(\alpha)=\operatorname{diag}\left(\alpha^{r_{1}}, \ldots, \alpha^{r_{n}}\right)$ for positive real constants $r_{i}$ such that

$$
h(G(\alpha) y, w)=\alpha^{d} G(\alpha) h(y, w)
$$

For example, for a function $h(y)=-y^{3}, G(\alpha)=\alpha$ and $d=2$.

Because we do not concern ourselves with homogeneous functions other than homogeneous-in-the-state functions, we simply call such a function homogeneous. Homogeneity will allow us to concentrate our analysis to the unit sphere. We denote the unit sphere in $\Re^{n}$ as the set $S^{n-1}$.

We now state two assumptions about the structure of (1).

Assumption 1: For each $i \in Q, g_{i}$ is a continuous, degree$\left(d_{i}+1\right)$ homogeneous function with $G(\alpha)=\alpha I$ for some real $d_{i} \geq 0$.

Assumption 2: For fixed $i, y\left(\tau, t_{0}, i\right)$ is continuous over the pair $\left(\tau, y_{0}\right)$.

C. Discrete-Time (DT) Switched Systems: Definitions and Notations

Because the controller will require switching logic to compute $i(\tau)$, it is practical to consider implementing it in discrete time. To this end, we examine DT systems with dynamics that arise from a time-discretization of (1) in which $g_{i}$ satisfy Assumption 1 .

In this paper, we apply a special state-dependent sampling period $T: \Re^{n} \times Q \rightarrow \Re^{+}$that will yield convenient properties for the resulting DT system, properties that a constant sampling period would not give us otherwise.

A DT system constructed using a variable sampling time $T$ is formally defined as follows. Let $t$ be an integer, let $i(t)$ be a DT input (i.e., $i=(i(0), i(1), i(2), \ldots)$ ), and let $x$ be the DT state. The sampling period at time $t$ is given by $T(x(t), i(t))$, and the DT system is

$$
x(t+1)=y(T(x(t), i(t)), x(t), i(t))
$$

Essentially, $x(t+1)$ is the value of the CT trajectory sampled at time $T(x(t), i(t))$ starting from the initial state $x(t)$ with the mode fixed to $i(t)$ over the time interval. For convenience, we denote the DT dynamics by $f_{i}(x)=$ $y(T(x, i), x, i)$ so that

$$
x(t+1)=f_{i(t)}(x(t))
$$

Now, for computational reasons, we are interested in having the DT system satisfy

$$
f_{i}(\alpha x)=\alpha f_{i}(x)
$$

for all modes $i$. We now provide a sampling time that yields this property.

Proposition 1: For a positive constant $T_{0}$, the statedependent sampling period given by $T(x, i)=T_{0}\|x\|^{-d_{i}}$ yields a continuous, degree- 1 homogeneous function $f_{i}$ for all $x$ and $i$.

We call $T_{0}$ the base sampling period.

In the remainder, $\tau$ is the CT time variable, $t$ is the DT time variable, $y$ is the CT state, $x$ is the DT state, and $i$ is the input in both settings.

\section{Objectives}

The remainder of this paper will be focused on determining conditions under which (1) can be sampled quickly enough to yield a DT system that is asymptotically controllable. Formally, we want to find a base sampling period $T_{0}$ and a DT feedback control law $u$ so that

$$
\begin{aligned}
x(t+1) & =f_{i(t)}(x(t)) \\
i(t+1) & =u(x(t), i(t))
\end{aligned}
$$


is stable. Note that, in this formulation, the mode $i$ is actually a system state, but we do not treat it as a state in our definition of stability and, rather, consider only the convergence of $x$.

\section{The VAlue FunCtion AND DynAmiC PROGRAMMING}

In this section, we present some basic definitions and results related to dynamic programming. We will use dynamic programming to construct optimal controllers for special cost functions that will guarantee feedback stabilizability.

\section{A. The CT and DT Value Functions}

In order to compute a stabilizing DT feedback law, we use a cost function for the CT system that will eventually be approximated in discrete time to yield a stabilizing control law.

Define the CT cost function $J\left(y_{0}, i\right)$ as

$$
\begin{aligned}
& J\left(y_{0}, i\right) \\
& \quad=\sum_{k=0}^{\infty}\left[\int_{\Delta_{k}}\left\|y\left(\tau, y_{k}, i_{k}\right)\right\|^{2+d_{i_{k}}} d \tau+\left\|y_{k}\right\|^{2} K_{i\left(\tau_{k}^{-}\right) i\left(\tau_{k}\right)}\right]
\end{aligned}
$$

where $\Delta_{k}$ and $y_{k}$ are the $k^{t h}$ dwell time and switching state respectively (as defined in Section II-A), and the switchingcost constants $K_{m n}$ are positive for $m \neq n$ and zero otherwise.

Optimizing over all switching laws $i$ with initial mode $i_{0}$, we obtain the $C T$ value function,

$$
J_{i_{0}}^{*}\left(y_{0}\right)=\inf _{\left\{i \mid i(0)=i_{0}\right\}} J\left(y_{0}, i\right)
$$

Now, define the DT cost function as

$$
\begin{aligned}
V\left(x_{0}, i\right) & =\sum_{t=0}^{\infty} L(x(t), i(t), i(t+1)) \\
& =\sum_{t=0}^{\infty}\left[T_{0}\|x(t)\|^{2}+\|x(t)\|^{2} K_{i(t) i(t+1)}\right]
\end{aligned}
$$

where $T_{0}$ is the base sampling period. Similarly, define the DT value function as

$$
V_{i_{0}}^{*}(x)=\inf _{\left\{i \mid i(0)=i_{0}\right\}} V_{i_{0}}(x, i)
$$

\section{B. The Bellman Equation and the DT Value Function}

In this section, we discuss a method for approximating $J_{i_{0}}^{*}$ and $V_{i_{0}}^{*}$.

Consider a general degree-1 homogeneous, DT switched system with control inputs $i$ and $w$,

$$
x(t+1)=\bar{h}_{i(t)}(x(t), w(t))
$$

and let $W \subset \Re^{m}$ be such that $w(\tau) \in W$. We consider a DT system with an additional input $w$ because we will eventually transform the CT switched system into a DT switched system with an additional input.
The computation of an optimal control law is tantamount to the computation of the value function $\bar{V}_{i_{0}}^{*}$ for all $i_{0} \in Q$, which satisfies Bellman's Equation

$$
\bar{V}_{i_{0}}^{*}(x)=\inf _{\{j, w\}}\left\{\bar{V}_{j}^{*}\left(\bar{h}_{i_{0}}(x, w)\right)+\bar{L}\left(x, w, i_{0}, j\right)\right\}
$$

where $\bar{L}$ is the incremental cost and is a function of the state, mode, and inputs. For ease, from here on we write $\bar{V}_{i}^{*}$ instead of $\bar{V}_{i_{0}}^{*}$, where in this case $i$ is understood to be a scalar in $Q$.

Now, let $\bar{h}_{i}$ and $\bar{L}$ satisfy the following assumptions.

Assumption 3: $\bar{h}_{i}$ is bounded over $S^{n-1} \times W$ (and, therefore, contained within a compact set).

Assumption 4: $\bar{L}$ is positive-definite, degree-2 homogeneous in $x$, and $\bar{L}(x, w, i, j) \geq \bar{L}(x, 0, i, j)$ for all $x, w, i, j$.

Under Assumption 4, it is easy to show that $V_{i}^{*}$ is degree-2 homogeneous.

If the value function $\bar{V}_{i}^{*}$ is known, the optimal policy can be computed through an evaluation of the expression

$$
\left(j^{*}(x, i), w^{*}(x, i)\right) \in \underset{\{j, w\}}{\operatorname{argmin}}\left\{\bar{V}_{j}^{*}\left(\bar{h}_{i}(x, w)\right)+\bar{L}(x, w, i, j)\right\}
$$

if the minimum exists.

In general, it is impossible to determine the value function analytically. On the other hand, a numerical approximation of the value function can be obtained by applying an algorithm called value iteration. In value iteration, successivelyimproving approximations to the value function are computed iteratively in the following manner: pick some $\bar{V}_{i}^{0}$ on $\Re^{n}$ and compute the sequence $\left(\bar{V}_{i}^{1}, \bar{V}_{i}^{2}, \ldots\right)$ iteratively by the relation

$$
\bar{V}_{i}^{k+1}(x)=\inf _{\{j, w\}}\left\{\bar{V}_{j}^{k}\left(\bar{h}_{i}(x, w)\right)+\bar{L}(x, w, i, j)\right\}
$$

For convenience, we always set the initial condition $\bar{V}_{i}^{0}=0$, which results in $\left(\bar{V}_{i}^{k}\right)_{k}$ being a monotonically increasing sequence of functions bounded by $\bar{V}_{i}^{*}$. Denote $\bar{V}_{i}^{\infty}=$ $\lim _{k \rightarrow \infty} \bar{V}_{i}^{k}$. While it is not generally true that value iteration will converge to $\bar{V}_{i}^{*}$, there are certain assumptions that may be imposed to guarantee the convergence of value iteration. In particular, the results of this paper make heavy use of a convergence result given in [1], which we restate here in a form more amenable to our framework.

Proposition 2: If $\bar{V}_{j}^{*}\left(\bar{h}_{i}(x, w)\right) \leq \gamma \bar{L}(x, w, i, j)$ holds uniformly for some $\gamma<\infty$ and if $\bar{V}_{i}^{*}$ is bounded over a compact set $E$, then $\left(\bar{V}_{i}^{k}\right)_{k}$ converges uniformly to $\bar{V}_{i}^{*}$ over E.

The two following useful results stem from an application of Proposition 2.

Lemma 1: If, for each $k$, (4) is minimized by some $j^{*}$ and $w^{*}$ and if $\bar{L}(\cdot, 0, i, j)$ is lower bounded by a positive constant over $S^{n-1}$ for all $i, j$, then $\bar{V}_{i}^{*}$ is equal to $\bar{V}_{i}^{\infty}$ if $\bar{V}_{i}^{\infty}$ is bounded over $S^{n-1}$.

Corollary 1: If $W$ is a compact set, $\bar{V}_{j}^{k}$ is continuous for all $k, \bar{h}_{i}(x, w)$ is continuous in $w$, and $\bar{L}(x, w, i, j)$ is continuous in $w$, then $\bar{V}_{i}^{*}$ is continuous and equal to $\bar{V}_{i}^{\infty}$ if $\bar{V}_{i}^{\infty}$ is bounded over $S^{n-1}$. 


\section{Stability under Discrete-Time Feedback}

To prove the existence of a base sampling period $T_{0}$ that yields an asymptotically controllable system, we must first prove that the CT value function is continuous so that we can treat the DT value function as an approximation of it.

\section{A. Continuity of the CT Value Function}

To simplify the proofs of this section, we apply a useful transformation that will generate a degree- 1 system having the same trajectories as (1). As in [3], let

$$
\dot{z}(\tau)=\tilde{g}_{i(\tau)}(z(\tau))=\|z(\tau)\|^{-d_{i(\tau)}} g_{i(\tau)}(z(\tau))
$$

Under suitable choices for each switching law, both (1) and (5) generate the same trajectories, but (5) is degree-1 homogeneous by this time scaling of (1).

If we define the cost function $\tilde{J}$ for system (5) as

$$
\begin{aligned}
& \tilde{J}\left(z_{0}, i\right) \\
& \quad=\sum_{k=0}^{\infty}\left[\int_{\Delta_{k}}\left\|z\left(\tau, z_{k}, i_{k}\right)\right\|^{2} d \tau+\left\|z_{k}\right\|^{2} K_{i\left(\tau_{k}^{-}\right) i\left(\tau_{k}\right)}\right]
\end{aligned}
$$

with $\tilde{J}_{i_{0}}^{*}$ is defined similarly as

$$
\tilde{J}_{i_{0}}^{*}\left(y_{0}\right)=\inf _{\left\{i \mid i(0)=i_{0}\right\}} \tilde{J}\left(y_{0}, i\right)
$$

then it can be proven that $\tilde{J}_{i}^{*}=J_{i}^{*}$. Therefore, from here on, we can assume without loss of generality that $d_{i}=1$ for all $i$ in (1). The remaining results will still hold for any set of $d_{i}^{\prime} s$ as long as the CT system is sampled using $T(x, i)$. Note that assuming $d_{i}=1$ yields $T(x, i)=T_{0}$, a constant.

First, we seek to show that $J_{i}^{*}$ is continuous by leveraging Corollary 1, but this result only applies to DT systems. In order to apply it to $J_{i}^{*}$, we relate $J_{i}^{*}$ to a DT Bellman equation, using the time until the subsequent switch as a control input over which we minimize. To this end, we define a new DT system with dynamics given by

$$
h_{i}(x, \tau)=y(\tau, x, i)
$$

Basically, $h_{i}$ is the sampled dynamics of (1) for a "sampling period" $\tau$, though we actually treat $\tau$ as an input. Note that $h_{i}\left(x, T_{0}\right)=f_{i}(x)$. Also, define

$$
l(x, \tau, i, j)=\int_{0}^{\tau}\|y(\gamma, x, i)\|^{2} d \gamma+\|y(\tau, x, i)\|^{2} K_{i j}
$$

as the sampled cost. The cost essentially represents the cost of allowing (1) to evolve in a fixed mode $i$ for $\tau$ time units, after which time the system changes to mode $j$.

If we treat $i$ and $\tau$ as control inputs, we have a degree-1 homogeneous DT system

$$
x(t+1)=h_{i(t)}(x(t), \tau(t))
$$

By substitution and by optimality, we can express $J_{i}^{*}$ by

$$
J_{i}^{*}(x)=\inf _{\left\{j, 0 \leq \tau \leq T_{0}\right\}}\left\{J_{j}^{*}\left(h_{i}(x, \tau)\right)+l(x, \tau, i, j)\right\}
$$

In essence, all we have done is split-up the expression of the value function by the switching times, which is possible by optimality. We can now express $J_{i}^{*}$ as the limit of the value iteration sequence $\left(J_{i}^{k}\right)_{k}$ where

$$
J_{i}^{k+1}(x)=\inf _{\left\{j, 0 \leq \tau \leq T_{0}\right\}}\left\{J_{j}^{k}\left(h_{i}(x, \tau)\right)+l(x, \tau, i, j)\right\}
$$

With these relationships, we are lead to the following important theorem.

Theorem 1: System (1) is asymptotically controllable if and only if $J_{i}^{*}$ is continuous.

Remark 1: It is important to note that the proof of continuity relies on $l(x, \tau, i, j)$ being positive for all $t$ if $i \neq j$. This condition is made possible by having positive switching costs $K_{i j}$. If $K_{i j}=0$ for some $i \neq j$, then $l(x, 0, i, j)=0$, and the boundedness condition of Proposition 2, which is used in the proof, would not hold.

\section{B. Discrete-Time Stabilization}

To prove the existence of a DT stabilizing control law, we use value iteration to generate a sequence of functions that converge to the DT value function while being bounded by $J_{i}^{*}$, which essentially bounds $V_{i}^{*}$ and proves the existence of control laws that yield a finite cost. We begin with the following convergence result.

Proposition 3: If $V_{i}^{\infty}$ is bounded over $S^{n-1}$, then $V_{i}^{*}=$ $V_{i}^{\infty}$ and $V_{i}^{*}$ is continuous.

We now state the main results of this paper.

Theorem 2 (Approximation of the CT value function): If (1) is asymptotically controllable, then for each $\epsilon>0$, there exists a positive time $\bar{T}_{0}$ such that for all base sampling periods $T_{0} \leq \bar{T}_{0},\left|J_{i}^{*}-V_{i}^{*}\right|<\epsilon$ over $S^{n-1}$.

Corollary 2 (Stability of the CT system via DT control): There exists a positive base sampling period $T_{0}$ such that (1) is asymptotically stable using the DT control law

$$
u^{*}(x, i) \in \underset{j}{\operatorname{argmin}}\left\{V_{j}^{*}\left(f_{i}(x)\right)+L(x, i, j)\right\}
$$

\section{Approximating the Value Function}

In this section, we present an algorithm for practically constructing a stabilizing DT feedback controller by approximating $V_{i}^{*}$.

\section{A. Value Iteration over a Finite Set}

By (3) and by the homogeneity of the functions $V_{i}^{*}, f_{i}$, and $L$, we have the following relationship for all $x \in S^{n-1}$

$$
\left.V_{i}^{*}(x)=\min _{j}\left\{\| f_{i}(x)\right) \|^{2} V_{j}^{*}\left(\frac{f_{i}(x)}{\left\|f_{i}(x)\right\|}\right)+L(x, i, j)\right\}
$$

where if $x=0$, we define $\|x\|^{2} V_{i}^{*}\left(\frac{x}{\|x\|}\right)=0$.

We now extend the relationship in (6) to the value iteration algorithm. For all $x \in S^{n-1}$, define the sequence of functions $\left(V_{i}^{k}\right)$ by

$$
V_{i}^{k+1}(x)=\min _{j}\left\{\left\|f_{i}(x)\right\|^{2} V_{j}^{k}\left(\frac{f_{i}(x)}{\left\|f_{i}(x)\right\|}\right)+L(x, i, j)\right\}
$$

Though (7) allows us to express $\left(V_{i}^{k}\right)_{k}$ as a sequence of functions only over the compact set $S^{n-1}$, a brute-force computation using (7) is still impractical. We now consider 
the implications of quantizing $S^{n-1}$ in order to practically compute an approximation to $V_{i}^{*}$.

First, we define our quantization function. For some $\delta>$ 0 , let $\hat{S}_{\delta}^{n-1}$ be a finite subset of $S^{n-1}$ such that for each $x \in S^{n-1}$, there is an approximating state $\hat{x} \in \hat{S}_{\delta}^{n-1}$ that is "close" to $x$ in the sense that $\|x-\hat{x}\|<\delta$. Define the quantization function $\Theta_{\delta}: \Re^{n} \rightarrow \Re^{n}$ by

$$
\Theta_{\delta}(x) \in\|x\| \underset{\hat{x} \in \hat{S}_{\delta}^{n-1}}{\operatorname{argmin}}\left\{\left\|\hat{x}-\frac{x}{\|x\|}\right\|\right\}
$$

Using homogeneity, $\Theta_{\delta}$ (which is degree-1 homogeneous) is able to generate an uncountable set of approximation points in $\Re^{n}$. From here on, we drop the $\delta$ subscript notation.

We now define a new value iteration over the set $\hat{S}^{n-1}$. For all $x \in \hat{S}^{n-1}$, define the sequence $\left(\hat{V}_{i}^{k}\right)$ by

$$
\begin{aligned}
\hat{V}_{i}^{k+1}(x)= & \min _{j}\left\{\hat{V}_{j}^{k}\left(\Theta\left(f_{i}(x)\right)\right)+L(x, i, j)\right\} \\
= & \min _{j}\left\{\left\|f_{i}(x)\right\|^{2} \hat{V}_{j}^{k}\left(\Theta\left(\frac{f_{i}(x)}{\left\|f_{i}(x)\right\|}\right)\right)\right. \\
& +L(x, i, j)\}
\end{aligned}
$$

Because $\Theta \circ f_{i}$ is degree- 1 homogeneous and bounded over $S^{n-1}$ and since the minimizer $j^{*}$ of (8) exists, we have by Lemma 1 that (8) converges to its cooresponding value function $\hat{V}_{i}^{*}$. Furthermore, since the computation is performed over a finite set, we can compute $\hat{V}_{i}^{*}$ using the following linear program [4], [7]

$$
\begin{aligned}
& \max \sum_{i \in Q, x \in \hat{S}^{n-1}} \hat{V}_{i}^{*}(x) \text { subject to } \\
& \quad \hat{V}_{i}^{*}(x) \leq\left\|f_{i}(x)\right\|^{2} \hat{V}_{j}^{*}\left(\Theta\left(\frac{f_{i}(x)}{\left\|f_{i}(x)\right\|}\right)\right)+L(x, i, j) \\
& \quad \text { for all } i, j \in Q \text { and } x \in \hat{S}^{n-1}
\end{aligned}
$$

where $\Theta\left(\frac{f_{i}(x)}{\left\|f_{i}(x)\right\|}\right) \in \hat{S}^{n-1}$.

The continuity results derived earlier come into play once we begin to consider the application of approximation states. If $V_{j}^{*}$ is $\delta$ - $\epsilon$ uniformly continuous over $S^{n-1}$, then for all $x \in S^{n-1}$, we have the following inequalities.

$$
V_{j}^{*}\left(f_{i}(x)\right) \lesseqgtr\left\|f_{i}(x)\right\|^{2}\left[V_{j}^{*}\left(\Theta\left(\frac{f_{i}(x)}{\left\|f_{i}(x)\right\|}\right)\right) \pm \epsilon\right]
$$

This relationship allows us to approximate the value function.

Proposition 4: If $V_{i}^{*}$ is continuous, then for each $\epsilon>0$, there exists a $\delta$ such that $\left|\hat{V}_{i}^{*}-V_{i}^{*}\right|<\epsilon$ over $\hat{S}^{n-1}$.

\section{B. Stability and Performance}

Given $\hat{V}_{i}^{*}(x)$, define the control law $u$ as

$$
u(x, i) \in \underset{j}{\operatorname{argmin}}\left\{\hat{V}_{j}^{*}\left(\Theta\left(f_{i}(x)\right)\right)+L(x, i, j)\right\}
$$

which exists. Clearly, system (2) subject to (9) is a degree-1 homogeneous system.

Now, to prove stability, we show the closed-loop system yields a finite cost

$$
\begin{aligned}
\tilde{V}_{i}(x) & =\sum_{t=0}^{\infty} L(x(t), i(t), u(x(t), i(t))) \\
& =\tilde{V}_{u(x, i)}\left(f_{i}(x)\right)+L(x, i, u(x, i))
\end{aligned}
$$

To derive conditions under which $\tilde{V}_{i}$ is bounded, we leverage the structure of (6) and compute a bound for the performance (and, hence, establish a certificate for stability) using value iteration. To this end, we define the sequence of functions $\left(\tilde{V}_{i}^{k}(x)\right)_{k}$ by

$$
\tilde{V}_{i}^{k+1}(x)=\tilde{V}_{u(x, i)}^{k}\left(f_{i}(x)\right)+L(x, i, u(x, i))
$$

Clearly, $\lim _{k \rightarrow \infty} \tilde{V}_{i}^{k}(x)$ exists and is equal to $\tilde{V}_{i}(x)$.

We now state the main performance and stability results of this section.

Lemma 2: If $V_{i}^{*}$ is continuous, then for each $\epsilon>0$, there exists a $\delta$ such that $\left|V_{i}^{*}-\tilde{V}_{i}\right|<\epsilon$ over $S^{n-1}$, and, hence, (2) is asymptotically stable.

Finally, for convenience, we summarize the results of this paper with the following theorem.

Theorem 3: System (1) is asymptotically controllable if and only if there exists a time $T_{\max }$ and a spacing $\delta_{\max }$ such that system (2) with $u$ given by (9) is asymptotically stable for all base sampling periods $T_{0} \leq T_{\max }$ and all quantization spacings $\delta<\delta_{\max } . u$ stabilizes (1) in discrete time, and the closed-loop cost $\tilde{V}_{i}$ may be made arbitrarily close $J_{i}^{*}$.

\section{Lipschitz Special Case}

Of course, in general, we do not know the $\delta$ - $\epsilon$ relationship for $V_{i}^{*}$, and so the results above only assert the existence of a level of approximation that provide these benefits. If we strengthen our assumptions about $L$ and $f_{i}$, though, we compute an upper bound for $\delta$ to offer a prescribed $\epsilon$.

Proposition 5: If $\{L(\cdot, \cdot, i, j)\}_{i, j}$ and $\left\{f_{i}\right\}_{i}$ are Lipschitz functions over $S^{n-1}$ with respective Lipschitz constants $\zeta$ and $\eta<1$, then $V_{i}^{*}$ is Lipschitz over $S^{n-1}$ with a Lipschitz constant $\frac{\zeta}{1-\eta}$.

It is noteworthy that the constraint on $\eta$ translates into the requirement each $f_{i}$ is a contraction.

\section{Simulations}

The example comes from a slight modification of the example switched system from [8]. The dual-mode switched system is given by

$$
g_{1}(y)=\left[\begin{array}{c}
0.1 y_{1}^{3}-y_{2}^{3} \\
10 y_{1}^{3}+0.1 y_{2}^{3}
\end{array}\right], g_{2}(y)=\left[\begin{array}{c}
0.1 y_{1}-10 y_{2} \\
y_{1}+0.1 y_{2}
\end{array}\right]
$$

where $y_{1}$ and $y_{2}$ are the components of the vector $y$. Both $g_{1}$ and $g_{2}$ are unstable systems that "spiral" away from the origin. Note that $g_{1}$ is degree- 3 homogeneous while $g_{2}$ is degree-1 homogeneous.

To construct a DT stabilizing control, we use a base sampling time of $0.1 \mathrm{~s}$ and a quantization spacing of 0.1 radians along a semi-sphere ${ }^{1}$, yielding 32 approximation states. Finally, we use the incremental cost function $L(x, i, j)=$ $\|x\|^{2}+\|x\|^{2} K_{i j}$ where $K_{i j}=1$ for $i \neq j$.

The optimal control laws $u$ (as given by (9)) are plotted in Figure 1 as a function of angle because they are independent of the magnitude of $y$.

Finally, Figure 2 shows a plot of CT closed-loop trajectories resulting from an initial state $y_{0}=(1,0)$ and $i_{0}=1$.

\footnotetext{
${ }^{1}$ By homogeneity, we need not consider the entire sphere.
} 

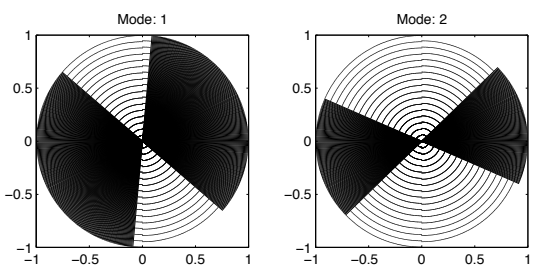

Fig. 1. Plot of $u$ for each initial mode. As $u$ is a degree- 0 homogeneous function, it is just a function of the angle in the phase space of $x$. Striped regions represent where $u$ takes a value of 1 , and solid regions represent where $u$ takes a value of 2
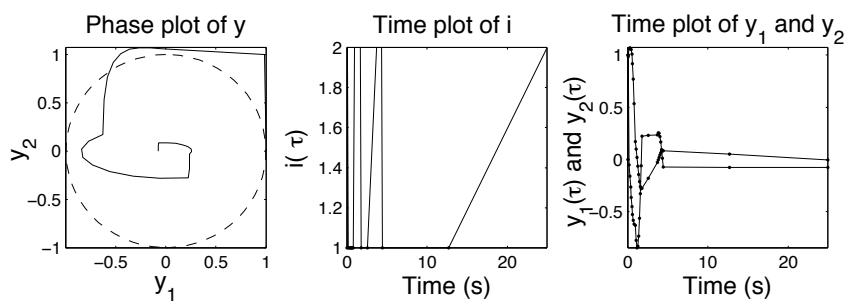

Fig. 2. Simulation of the closed-loop systems from the initial pair $y_{0}=$ $(1,0)$ and $i_{0}=1$.

Notice that although the system is executed for 30 time samples, the simulations take nearly 30 seconds to complete, despite a 0.1 base sampling period. This is because as the trajectory approaches the origin, $g_{1}$ reacts far more slowly, a consequence of using $T(x, 1)=\|x\|^{-2} T_{0}$, which approaches $\infty$ as $\|x\| \rightarrow 0$.

\section{CONCLUSIONS AND FUTURE WORK}

In this paper, a framework for constructing stabilizing discrete-time state-feedback controllers for continuous-time switched homogeneous systems was presented. Homogeneity and a state-dependent sampling period were leveraged to reduce the difficulty of computing a nearly optimal controller to a linear program over the unit sphere.

We believe much can be gained from applying the theoretical methodology to practical problems. A particular application of interest is determining if a switched system is stabilizable under all possible switching sequences. Modifications to our framework may be able to be address this problem by searching for the optimal unstable trajectory. An additional extension of interest is the control of nonlinear systems that can be locally approximated by homogeneous systems at a set of equilbrium points. If the number of such points is sizable, our approach may be able to be used in conjunction with another algorithm to provide a means for maneuvering through the state space, similar to the approach taken in [9].

\section{ACKNOWLEDGMENTS}

The authors wish to thank Dr. Davor Hrovat for his initiation of this joint project. This research is sponsored by Ford Research and Advanced Engineering, Ford Motor Company.

\section{REFERENCES}

[1] A. Rantzer, "Approximate dynamic programming in switching systems," in Proceedings of 44th Conference on Decision and Control and European Control Conference, Dec. 2005, pp. 1391-1396.

[2] B. Lincoln and A. Rantzer, "Relaxing dynamic programming," IEEE Trans. Autom. Control, vol. 51, no. 8, pp. 1249-1260, Aug. 2006.

[3] L. Grune, "Homogeneous state feedback stabilization of homogeneous systems," in Proceedings of the 39th IEEE Conference on Decision and Control, vol. 4, December 2000, pp. 3409-3414.

[4] M. Rinehart, M. Dahleh, D. Reed, and I. Kolmanovsky, "Suboptimal control of switched systems with an application to the disc engine," IEEE Trans. Control Syst. Technol. (to appear), 2007.

[5] M. Rinehart, M. Dahleh, and I. V. Kolmanovski, "Optimal control of swtiched homogeneous systems," IEEE Trans. Autom. Control (aubmitted), 2007.

[6] F. Clarke, Y. Ledyaev, E. Sontag, and A. Subbotin, "Asymptotic controllability implies feedback stabilization," IEEE Trans. Autom. Control, vol. 45, no. 1, pp. 165-176, 1999.

[7] D. Bertsekas, Dynamic Programming and Optimal Control (vol. I), 3rd ed. Nashua, NH: Athena Scientific, 2005.

[8] M. Branicky, "Multiple lyapunov functions and other analysis tools for switched and hybrid systems," IEEE Trans. Autom. Control, vol. 43, no. 4, pp. 475-482, Apr. 1998.

[9] M. W. McConley, "A computationally efficient lyapunov-based procedure for control of nonlinear systems with stability and performance guarantees," Ph.D. dissertation, Mass. Inst. of Tech. Cambridge, MA, May 1997. [Online]. Available: http://dspace.mit.edu

[10] M. Branicky, V. Borkar, and S. Mitter, "A unified framework for hybrid control: Model and optimal control theory," IEEE Trans. Autom. Control, vol. 43, no. 1, pp. 31-45, Jan. 1998.

[11] N. H. El-Farra and P. D. Christofides, "Switching and feedback laws for control of constrained switched nonlinear systems," in HSCC '02: Proceedings of the 5th International Workshop on Hybrid Systems: Computation and Control. London, UK: Springer-Verlag, 2002, pp. $164-178$.

[12] S. Hedlund and A. Rantzer, "Optimal control of hybrid systems," in Proceedings of the 38th IEEE Conference on Decision and Control, Dec. 1999, pp. 3972-3976.

[13] H. Ninomiya and H. Weinberger, "On p-homogeneous systems of differential equations and their linear perturbations," Applicable Analysis, vol. 85, no. 1-3, pp. 225-246, Jan.-Mar. 2006.

[14] L. Rosier, "Homogeneous lyapunov function for homogeneous continuous vector field," Systems and Control Letters, vol. 19, no. 6, pp. 467-473, Dec. 1992.

[15] W. Rudin, Principles of Mathematical Analysis. New York, NY: McGraw-Hill Inc., 1976.

[16] C. Seatzu, D. Corona, A. Giua, and A. Bemporad, "Optimal control of continuous-time switched affine systems," IEEE Trans. Autom. Control (submitted), 2006.

[17] Z. Sun and S. S. Ge, Switched Linear Systems: Control and Design. London, UK: Springer-Verlag, 2005.

[18] S. Tuna and A. Teel, "Regulating discrete-time homogeneous systems under arbitrary switching," in Proceedings of the 44th IEEE Conference on Decision and Control, Dec. 2005, pp. 2586-2591.

[19] X. Xu and P. Antsaklis, "Optimal control of switched systems based on a parameterization of switching instants," IEEE Trans. Autom. Control, vol. 49, no. 1, pp. 2-16, 2004. 